\title{
Impedances of Long Antennas in Air and in Dissipative Media
}

\author{
D. W. Gooch, ${ }^{2}$ C. W. Harrison, Jr., ${ }^{3}$ R. W. P. King, ${ }^{2}$ and T. T. Wu ${ }^{2}$
}

(Received January 4, 1963)

\begin{abstract}
Graphs are provided for the normalized impedance of center-driven cylindrical dipole antennas when immersed in air or in a dissipative medium. The electric half-length ranges from 1 to 100 for dipoles in air and from 1 to 19.7 for dipoles in a dissipative medium. Three ratios of radius of the antenna to wavelength have been used. The properties of the medium are expressed in terms of the ratio $\alpha / \beta$ in the range from zero to one where $\beta$ and $\alpha$ are, respectively, the real and imaginary parts of the complex propagation constant $k$.
\end{abstract}

\section{Introduction}

Antennas completely immersed in a dissipative medium that is characterized by the constitutive parameters $\sigma, \epsilon$, and $\mu$ have been a subject of interest for many years. Most investigations were concerned primarily with the electromagnetic fields of idealized dipole sources and a very extensive literature exists on this subject. Studies of the circuit and field properties of insulated antennas [Moore, 1951] have been based on a transmission-line approximation that is useful for media that are rather highly conducting so that $(\sigma / \omega \epsilon)>>1$. The distribution of current and the impedance characteristics of center-driven bare antennas of half-length $h$ and radius $a$ have been reported specifically for the electrically short antenna [King, Harrison, and Denton, 1961] with $\beta b \leq 0.3$, $0 \leq(\sigma \mid \omega \epsilon) \leq \infty$; and for the half-wave dipole [King and Harrison, 1960] with $\beta h=\pi / 2,0 \leq(\sigma / \omega \epsilon) \leq 0.4$. Approximate formulas have also heen obtained [King, 1962; King and Iizuka, 1963] for cylindrical antennas in the ranges $\beta h<\pi, 0 \leq(\sigma / \omega \epsilon) \leq \infty$.

Extensive investigations of the circuit properties of electrically long antennas have been concluded recently. These include a theoretical study by T. T. $\mathrm{Wu}$ [1961] which provides an asymptotic formula, derived by the Wiener-Hopf method, for the impedance of a long cylindrical antenna center driven by a delta-function generator under the very general conditions, $(a / h)<<1,(a / \lambda)<<1, \beta h>1,0 \leq(\sigma / \omega \epsilon) \leq \infty$,

\footnotetext{
1 Supported in part by Joint Services Contract Nonr 1866(32) between Harvard University, the Office of Naval Research, the Signal Corps of the U.S. Army, and the U.S. Air Force; National Science Foundation Grant NSF-G20225; and the Sandia Corporation.

2 Gordon McKay Laboratory, Harvard University, Cambridge, Mass.

3 Sandia Corporation, Albuquerque, N. Mex.
}

and a complete tabulation of the impedance for four values of $a / \lambda$ in the range $1 \leq \beta h \leq 30$ when $\sigma=0$. Analytical difficulties associated with the idealized generator have been discussed in detail $[\mathrm{Wu}$ and King, 1959; King and Harrison, 1960, and King, 1962]. A careful series of experimental measurements of the impedance of long antennas in air [Tizuka, King, and Prasad, 1963] (which takes full account of terminal-zone effects and the relationship between impedances measured with an actual transmission line and those computed for a delta-function generator) confirms the accuracy of the theoretical results when $\beta h \geq \pi$ and shows them to be useful approximations even when $\beta h$ is as small as 1 .

\section{Antennas in Dissipative Media}

Since no complete quantitative data have been published of the impedance of bare antennas in dissipative media in the range $1 \leq \beta h \leq \infty$, $0 \leq(\sigma / \omega \epsilon) \leq \infty$ extensive computations have been made from Wu's formula. The purpose was to provide quantitatively useful information on the properties of antennas with a wide range of lengths and diameters when immersed in media ranging from air $\left(\epsilon_{r}=1, \sigma=0\right)$ through dry earth $\left(\epsilon_{r} \sim 4\right.$, $\left.\sigma \sim 10^{-5}\right)$, moist earth $\left(\epsilon_{r} \sim 10, \sigma \sim 10^{-3}\right)$ to salt water $\left(\epsilon_{r} \sim 80, \sigma \sim 4\right)$. Such information has obvious applications to subsurface communication, to the study of the properties of dissipative media, and to the theory of ground systems in general. Although the results apply specifically to an infinite medium, the impedance of an antenna is determined primarily by the adjacent medium especially when this is even moderately conducting. It follows that the impedance of an antenna in moist earth or salt 
water near an air interface is well approximated by the impedance of an antenna in an infinite medium if the distance to the surface is not too small. Measurements to determine the effect of such an interface will be reported in another paper.

Wu's formulas for the admittance $Y=G-i B$ and the impedance $Z=R-i X$ (for a time dependence $\left.e^{-i \omega t}\right)$ are [Wu, 1961]

$$
Y=\frac{1}{Z}=\frac{2 i k}{\omega \mu}(S+C U) \operatorname{mhos}
$$

where

$$
\begin{gathered}
C=-\frac{1}{2} \frac{\left(2 T-T^{\prime}\right) \sin k h-\left(2 S-S^{\prime}\right) \cos k h}{T^{\prime} \cos k h+S^{\prime} \sin k h} \\
U=-i\left(A_{2}-A_{3}\right) .
\end{gathered}
$$

The following quantities are involved in the definitions of $C$ and $U$ :

$$
\begin{gathered}
\gamma=0.57722, \quad \gamma^{\prime}=1.6449 \\
\Omega_{0}=\ln (\lambda / a)-\ln \pi-\gamma, \quad \Omega_{0}^{\prime}=\Omega_{0}-\ln 2 \\
A_{1}=\ln \left[1+\frac{\pi i}{\Omega_{0}^{\prime}}\right]+\frac{\pi^{2}}{12}\left[\frac{1}{\left(\Omega_{0}^{\prime}-\ln 2\right)^{2}}-\frac{1}{\left(\Omega_{0}^{\prime}-\ln 2+\pi i\right)^{2}}\right]
\end{gathered}
$$$$
\Omega_{2}=2 \Omega_{0}^{\prime}+\ln (2 k h)+\gamma-\frac{i \pi}{2}, \quad \Omega_{2}^{\prime}=\Omega_{2}+\ln 2
$$$$
\Omega_{3}=\Omega_{2}+2 \pi i, \quad \Omega_{3}^{\prime}=\Omega_{2}^{\prime}+2 \pi i
$$$$
A_{2}=\ln \left(\Omega_{3} / \Omega_{2}\right)+\frac{1}{2} \gamma^{\prime}\left[\Omega_{2}^{-2}-\Omega_{3}^{-2}\right]
$$$$
A_{2}^{\prime}=\ln \left(\Omega_{3}^{\prime} / \Omega_{2}^{\prime}\right)+\frac{1}{2} \gamma^{\prime}\left[\Omega_{2}^{\prime-2}-\Omega_{3}^{\prime-2}\right]
$$$$
A_{3}=\frac{-i}{2 k h} e^{2 i k h}\left|\frac{1}{\Omega_{2}}-\frac{1}{\Omega_{3}}\right|
$$$$
A_{3}^{\prime}=\frac{-i}{4 k h} e^{4 i k h}\left|\frac{1}{\Omega_{2}^{\prime}},-\frac{1}{\Omega_{3}^{\prime}}\right|
$$$$
S=\frac{1}{2}\left(-A_{1}+A_{2}+A_{3}\right), \quad S^{\prime}=\frac{1}{2}\left(-A_{1}+A_{2}^{\prime}+A_{3}^{\prime}\right)
$$

$T=\frac{1}{2} i\left(-A_{1}-A_{2}+A_{3}\right), \quad T^{\prime}=\frac{1}{2} i\left(-A_{1}-A_{2}^{\prime}+A_{3}^{\prime}\right)$.

The complex propagation constant of the medium in which the highly conducting antenna is immersed is given by

$$
k=\beta+i \alpha=\sqrt{\mu \epsilon(1+i p)}=k_{0} \sqrt{\mu_{r} \epsilon_{r}(1+i p)}
$$

where

$$
k_{0}=\sqrt{\mu_{0} \epsilon_{0}}, \quad p=\frac{\sigma}{\omega \epsilon_{0} \epsilon_{r}} .
$$

Note that $k_{0}$ is the propagation constant for free space and $p$ is the loss tangent of the medium. The phase constant $\beta$ and attenuation constant $\alpha$ are conveniently expressed in terms of the real and imaginary parts of the function

where

$$
\sqrt{1 \pm i p}=f(p) \pm i g(p)
$$

$$
\begin{aligned}
& f(p)=\cosh ^{-1}\left(\frac{1}{2} \sinh ^{-1} p\right)=\sqrt{\frac{1}{2}\left(\sqrt{1+p^{2}}+1\right)} \\
& g(p)=\sinh ^{-1}\left(\frac{1}{2} \sinh ^{-1} p\right)=\sqrt{\frac{1}{2}\left(\sqrt{1+p^{2}}-1\right)} .
\end{aligned}
$$

These functions are readily available in the literature [King, 1945; Gooch, Harrison, King, and Wu, 1962]. With (18) and (19) the real and imaginary parts of $k$ are

$$
\begin{gathered}
\beta=k_{0} \sqrt{\epsilon_{r} \mu_{r}} f(p), \quad \alpha=k_{0} \sqrt{\epsilon_{r} \mu_{r}} g(p) \\
\alpha=0, \quad \beta=k_{0} .
\end{gathered}
$$

Owing to the complex intrinsic impedance

$$
\zeta=\frac{\omega \mu}{k}=\frac{\zeta_{0}}{\Delta(1-i \alpha / \beta)}
$$

where

$$
\zeta_{0}=\left(\mu_{0} / \epsilon_{0}\right)^{1 / 2}
$$

and

$$
\Delta=\sqrt{\frac{\boldsymbol{\epsilon}_{r}}{\mu_{r}}} f(p)
$$

which is a coefficient in the formula (1) for the admittance, it is not possible to provide universal curves of the impedance of antennas in dissipative media with $\beta h$ as variable and $a / \lambda$ and $\alpha / \beta$ as parameters in the manner familiar for antennas in air. The most general form in which the admittance and impedance can be presented is

$$
Y / \Delta=G / \Delta-i B / \Delta, Z \Delta=R \Delta-i X \Delta
$$

where the normalizing factor $\Delta$ is given in (22b). For antennas in air, $\Delta=1$; for antennas in perfect dielectrics $\sigma=0$ so that $p=0$ and $f(p)=1$ and $\Delta=$ $\sqrt{\boldsymbol{\epsilon}_{r} / \mu_{r}}$. When $\sigma \neq 0$ the actual impedance may be obtained from the normalized value $Z \Delta$ by dividing by $\Delta$ as evaluated from $(22 \mathrm{~b})$ with the aid of the tables [King, 1945; Gooch, Harrison, King, and Wu, 1962] of $f(p)$.

Normalized admittances and impedances have 
been computed for antennas with three different radii, viz,

$$
\frac{\beta a}{2 \pi}=\frac{a}{\lambda}=0.001191,0.003175,0.008496
$$

where $\lambda$ is the wavelength in the medium. The electrical half-lengths in dissipative media range from $\beta h=1$ to $\beta h=19.7$. The properties of the medium are expressed in terms of the ratio $\alpha / \beta=$ $g(p) / f(p)$ in the range from $\alpha / \beta=0$ (air) to $\alpha / \beta=1$ (salt water). The results of the computation are contained in the three families of impedance curves shown in figures 1, 2, and 3 for the three values of $a / \lambda$. They are available in complete tabular form in Gooch et al. [1962]. A short list of impedances is in the table. The effect of increasing the dimensionless ratio $\alpha / \beta$ on the resistance and reactance of an antenna as it is made longer is brought out very clearly in these figures. Note the rapid decrease in the antiresonant resistances near $\beta h=n \pi$ with $n$ integral as $\alpha / \beta$ is increased. This is shown best with $\beta h$ near $\pi$ in passing from the peak at $A$ for $\alpha / \beta=0$ to that at $I$ for $\alpha / \beta=1$. In figure 1 it is evident from

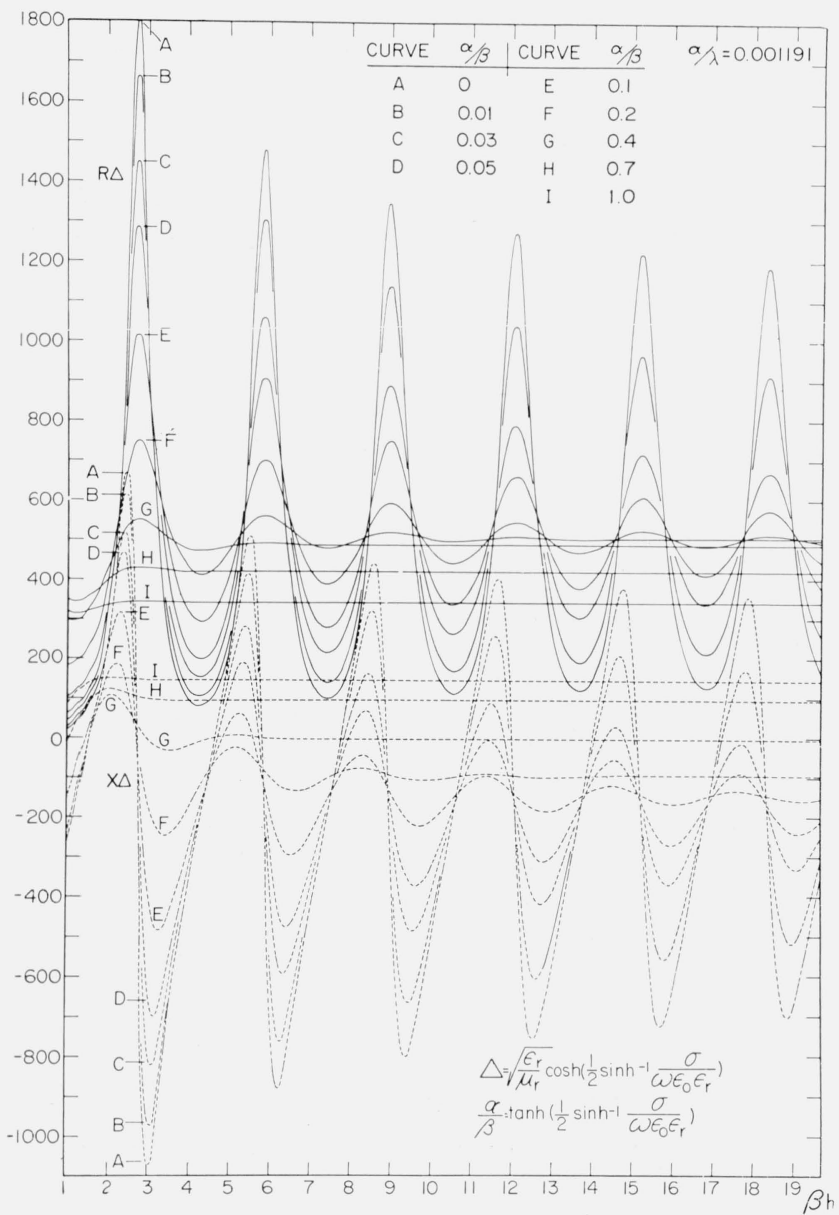

FIG. 1. Normalized resistance and reactance of dipole antennas in air and in dissipative media.

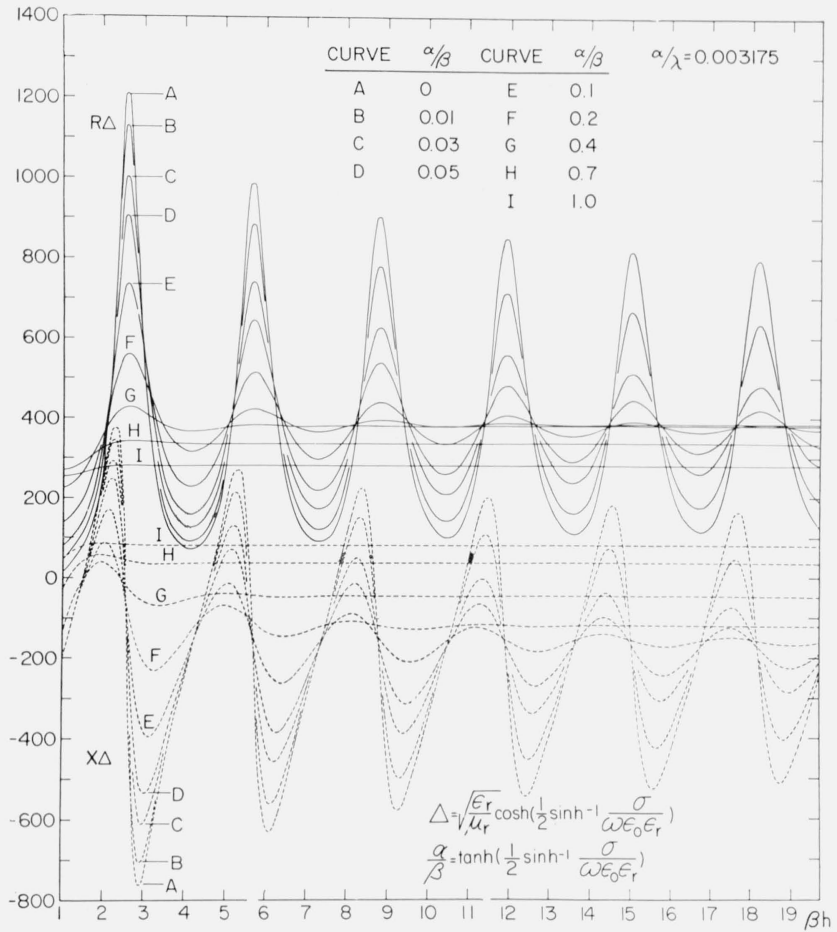

Fig. 2. Normalized resistance and reactance of dipole untennas in air and in dissipative media.

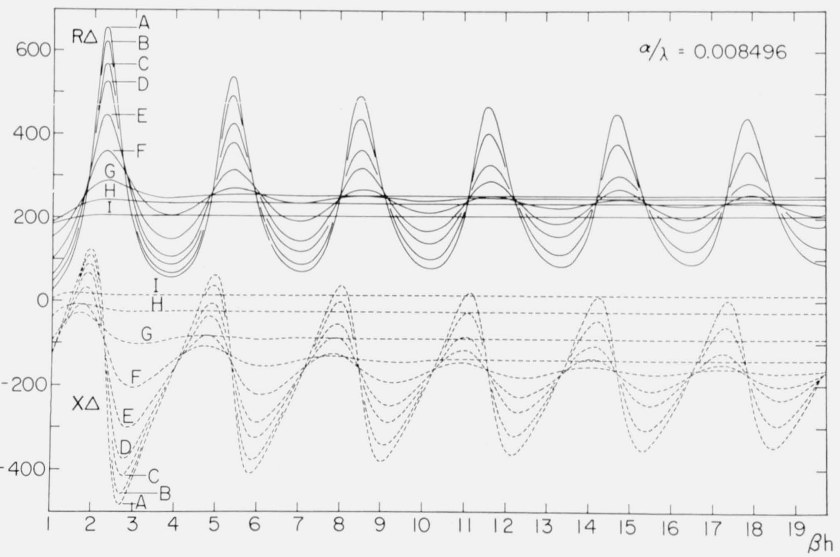

FIG. 3. Normalized resistance and reactance of dipole antennas in air and in dissipative media.

curves $F$ that with $\alpha / \beta=0.2, R \Delta$ and $X \Delta$ are essentially independent of further increases in length beyond $\beta h=12$, from curves $G$ with $\alpha / \beta=0.4$ this independence of length is seen to begin near $\beta h=6$, from curves $H$ with $\alpha / \beta=0.7$ the independence begins at $\beta h=3$. Finally for curve $I$ with $\alpha / \beta=1$ the impedance hardly changes for lengths greater than $\beta h=2$. It may be inferred that the current is negligible in those extensions of the conductor that have no effect on the impedance. 
Short table of the normalized impedance of a dipole in a dissipative medium $Z \Delta=R \Delta-i X \Delta$ for $a / \lambda=0.0031 \% 5$

\begin{tabular}{|c|c|c|c|c|c|c|c|c|}
\hline \multicolumn{3}{|c|}{$\beta h=1.5708$} & \multicolumn{2}{|c|}{2.0} & \multicolumn{2}{|c|}{2.3} & \multicolumn{2}{|c|}{2.6} \\
\hline$\frac{\alpha}{\beta}$ & $R \Delta$ & $-X \Delta$ & $R \Delta$ & $-X_{\Delta}$ & $R \Delta$ & $-X \Delta$ & $R \Delta$ & $-X_{\Delta}$ \\
\hline $\begin{array}{l}0.0 \\
.01 \\
.03 \\
.05 \\
.1 \\
.4 \\
1.0\end{array}$ & $\begin{array}{l}104 \\
111 \\
124 \\
137 \\
168 \\
282 \\
266\end{array}$ & $\begin{array}{l}-48.8 \\
-46.0 \\
-40.7 \\
-35.7 \\
-24.8 \\
-12.0 \\
-83.1\end{array}$ & $\begin{array}{l}304 \\
313 \\
327 \\
339 \\
358 \\
367 \\
277\end{array}$ & $\begin{array}{l}-277 \\
-263 \\
-237 \\
-213 \\
-160 \\
-37.5 \\
-85.4\end{array}$ & $\begin{array}{l}721 \\
710 \\
684 \\
658 \\
597 \\
412 \\
279\end{array}$ & $\begin{array}{l}-374 \\
-336 \\
-271 \\
-218 \\
-126 \\
-11.9 \\
-83.5\end{array}$ & $\begin{array}{r}1210 \\
1130 \\
1000 \\
905 \\
736 \\
427 \\
280\end{array}$ & $\begin{array}{r}232 \\
215 \\
189 \\
168 \\
132 \\
26.8 \\
-81.8\end{array}$ \\
\hline \multicolumn{3}{|c|}{$\beta h=2.9$} & \multicolumn{2}{|c|}{3.1416} & \multicolumn{2}{|r|}{3.5} & \multicolumn{2}{|c|}{3.8} \\
\hline$\frac{\alpha}{\beta}$ & $R \Delta$ & $-X \Delta$ & $R \Delta$ & $-X \Delta$ & $R \Delta$ & $-X \Delta$ & $R \Delta$ & $-X \Delta$ \\
\hline $\begin{array}{c}0.0 \\
.01 \\
.03 \\
.05 \\
.1 \\
.4 \\
1.0\end{array}$ & $\begin{array}{l}674 \\
676 \\
669 \\
654 \\
603 \\
417 \\
280\end{array}$ & $\begin{array}{c}764 \\
703 \\
599 \\
513 \\
359 \\
56.4 \\
-80.8\end{array}$ & $\begin{array}{l}337 \\
360 \\
396 \\
421 \\
451 \\
402 \\
280\end{array}$ & $\begin{array}{r}678 \\
646 \\
583 \\
524 \\
397 \\
68.2 \\
-80.5\end{array}$ & $\begin{array}{l}144 \\
166 \\
206 \\
240 \\
305 \\
381 \\
279\end{array}$ & $\begin{array}{c}476 \\
464 \\
438 \\
410 \\
340 \\
69.7 \\
-80.4\end{array}$ & $\begin{array}{l}87.7 \\
107 \\
144 \\
177 \\
246 \\
371 \\
279\end{array}$ & $\begin{array}{r}335 \\
330 \\
318 \\
304 \\
264 \\
62.7 \\
-80.5\end{array}$ \\
\hline \multicolumn{3}{|c|}{$\beta h=4.1$} & \multicolumn{2}{|c|}{4.4} & \multicolumn{2}{|c|}{4.7124} & \multicolumn{2}{|c|}{5.0} \\
\hline$\frac{\alpha}{\beta}$ & $R \Delta$ & $-X \Delta$ & $R \Delta$ & $-X \Delta$ & $R \Delta$ & $-X \Delta$ & $R \Delta$ & $-X \Delta$ \\
\hline $\begin{array}{c}0.0 \\
.01 \\
.03 \\
.05 \\
.1 \\
.4 \\
1.0\end{array}$ & $\begin{array}{c}72.2 \\
90.6 \\
126 \\
158 \\
227 \\
367 \\
279\end{array}$ & $\begin{array}{r}211 \\
210 \\
206 \\
202 \\
186 \\
53.6 \\
-80.6\end{array}$ & $\begin{array}{l}83.3 \\
105 \\
141 \\
173 \\
241 \\
368 \\
279\end{array}$ & $\begin{array}{c}87.9 \\
91.4 \\
97.8 \\
103 \\
111 \\
46.1 \\
-80.6\end{array}$ & $\begin{array}{l}144 \\
164 \\
201 \\
232 \\
289 \\
372 \\
279\end{array}$ & $\begin{array}{c}-50.4 \\
-38.3 \\
-15.2 \\
5.92 \\
46.6 \\
41.7 \\
-80.6\end{array}$ & $\begin{array}{l}272 \\
292 \\
321 \\
340 \\
364 \\
377 \\
279\end{array}$ & $\begin{array}{r}-188 \\
-159 \\
-106 \\
-61.0 \\
15.6 \\
40.8 \\
-80.6\end{array}$ \\
\hline \multicolumn{3}{|c|}{$\beta h=5.3$} & \multicolumn{2}{|c|}{5.7} & \multicolumn{2}{|r|}{6.1} & \multicolumn{2}{|c|}{6.2832} \\
\hline$\frac{\alpha}{\beta}$ & $R \Delta$ & $-X \Delta$ & $R \Delta$ & $-X_{\Delta}$ & $R \Delta$ & $-X_{\Delta}$ & $R \Delta$ & $-X \Delta$ \\
\hline $\begin{array}{c}0.0 \\
.01 \\
.03 \\
.05 \\
.1 \\
.4 \\
1.0\end{array}$ & $\begin{array}{l}572 \\
561 \\
534 \\
507 \\
455 \\
380 \\
279\end{array}$ & $\begin{array}{r}-270 \\
-209 \\
-116 \\
-51.2 \\
36.8 \\
42.3 \\
-80.6\end{array}$ & $\begin{array}{l}984 \\
883 \\
740 \\
645 \\
514 \\
382 \\
279\end{array}$ & $\begin{array}{r}238 \\
217 \\
190 \\
173 \\
148 \\
45.6 \\
-80.6\end{array}$ & $\begin{array}{l}532 \\
535 \\
525 \\
506 \\
459 \\
381 \\
279\end{array}$ & $\begin{array}{c}631 \\
561 \\
449 \\
368 \\
246 \\
48.0 \\
-80.6\end{array}$ & $\begin{array}{l}358 \\
381 \\
408 \\
419 \\
418 \\
380 \\
279\end{array}$ & $\begin{array}{r}594 \\
544 \\
456 \\
384 \\
262 \\
48.6 \\
-80.6\end{array}$ \\
\hline
\end{tabular}

\section{Very Long Antennas in Air}

A more complete range of impedances for long antennas in air is shown in figures $4 \mathrm{a}$, b where the electrical length is extended to $\beta h \approx 100$. For the range $\beta h \leq 4.0$ the impedances determined from the King-Middleton [King, 1956] theory are shown since they are more accurate in this range than the $\mathrm{Wu}$ theory [Wu, 1961] which, in turn, is more accurate for longer antennas. Note that for dipoles in air $\Delta=1$, so that the normalized and actual impedances coincide.

The graphs for the impedance of a dipole in air in figures 1,2 , and $3(\alpha=0)$ and in figures $4 \mathrm{a}, \mathrm{b}$ show that the oscillations in both $R$ and $X$, with increasing values of electrical length $\beta h$, decrease only very slowly in amplitude for any given $a / \lambda$. The limiting values of the admittance, as the length of the antenna approaches infinity at a fixed frequency $\omega$, are given by [King and Schmitt, 1962]

$$
\begin{gathered}
G=\frac{1}{\zeta_{0}}\left[\tan ^{-1} \frac{\pi}{\Omega_{0}}+6\left(\frac{\pi^{3} \Omega_{0}^{\prime}}{\left.\Omega_{0}^{\prime 2}+\pi^{2}\right)}\right]\right. \\
B=\frac{1}{2 \zeta_{0}}\left[\ln \left(1+\frac{\pi^{2}}{\Omega_{0}^{2}}\right)+\frac{\pi^{4}\left(3 \Omega_{0}^{\prime 2}+\pi^{2}\right)}{6 \Omega_{0}^{\prime 2}\left(\Omega_{0}^{\prime 2}+\pi^{2}\right)^{2}}\right]
\end{gathered}
$$

where $\zeta_{0}=120 \pi$ ohms. Approximate expressions when $k_{a}=2 \pi a / \lambda$ are sufficiently small so that

$$
\left(\frac{\pi}{\Omega_{0}}\right)^{2}<<1
$$

is satisfied are given by the leading terms in (24) and (25). They are

$$
G \doteq \frac{\pi}{\zeta_{0} \Omega_{0}}, B \doteq \frac{\pi^{2}}{2 \zeta_{0} \Omega_{0}^{2}}
$$

This formula for the conductance is essentially the same as that derived by Papas [1958]. The limiting value of the impedance corresponding to the admittance defined by (27) is

$$
Z \doteq \frac{\zeta_{0}}{\pi} \Omega_{0}+i \frac{\zeta_{0}}{2}
$$

so that

$$
\begin{gathered}
R \doteq \frac{\zeta_{0}}{\pi}\left[\ln \left(\frac{\lambda}{2 \pi a}\right)-0.5772\right] \\
X \doteq-\frac{\zeta_{0}}{2} \doteq-60 \pi \text { ohms }
\end{gathered}
$$

\section{Conclusion}

In conclusion it may be stated that accurate quantitative data have been provided for the impedance of bare cylindrical antennas over a very wide range of lengths when immersed in an arbitrary homogeneous and isotropic medium. Although computed specifically for a delta-function generator at the center of a dipole, the results are readily applied to antennas center driven from a two-wire line [King, 1955, 1956; Iizuka and King, 1962] or a monopole base driven over a ground screen by means of a coaxial line [King, 1955a, b, and 1956] if proper corrections are made for the differences in the terminal zones.

This paper was prepared at Harvard University by Dilla W. Gooch, R. W. P. King, and T. T. Wu. The computations upon which the graphical representations are based were carried out at the Sandia Corporation under the direction of C. W. Harrison, Jr. 

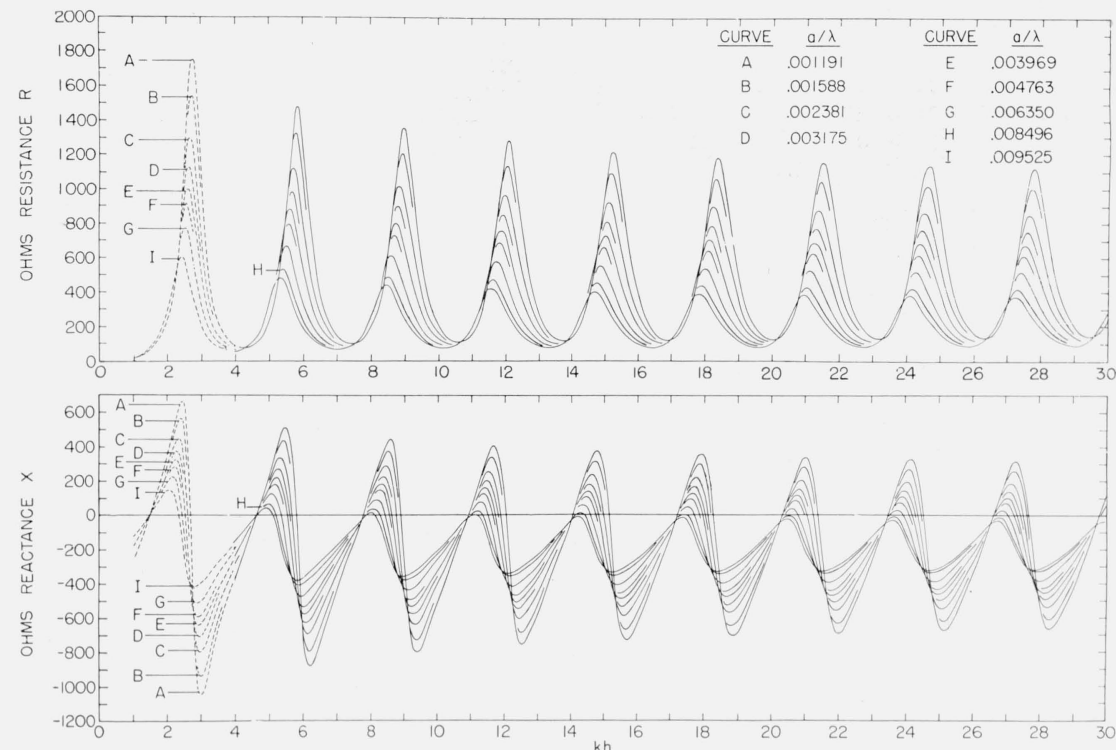

Fig. 4a. Resistance and reactance of cylindrical antenna.

- Wu

_..... King-Middleton 2d-order theory
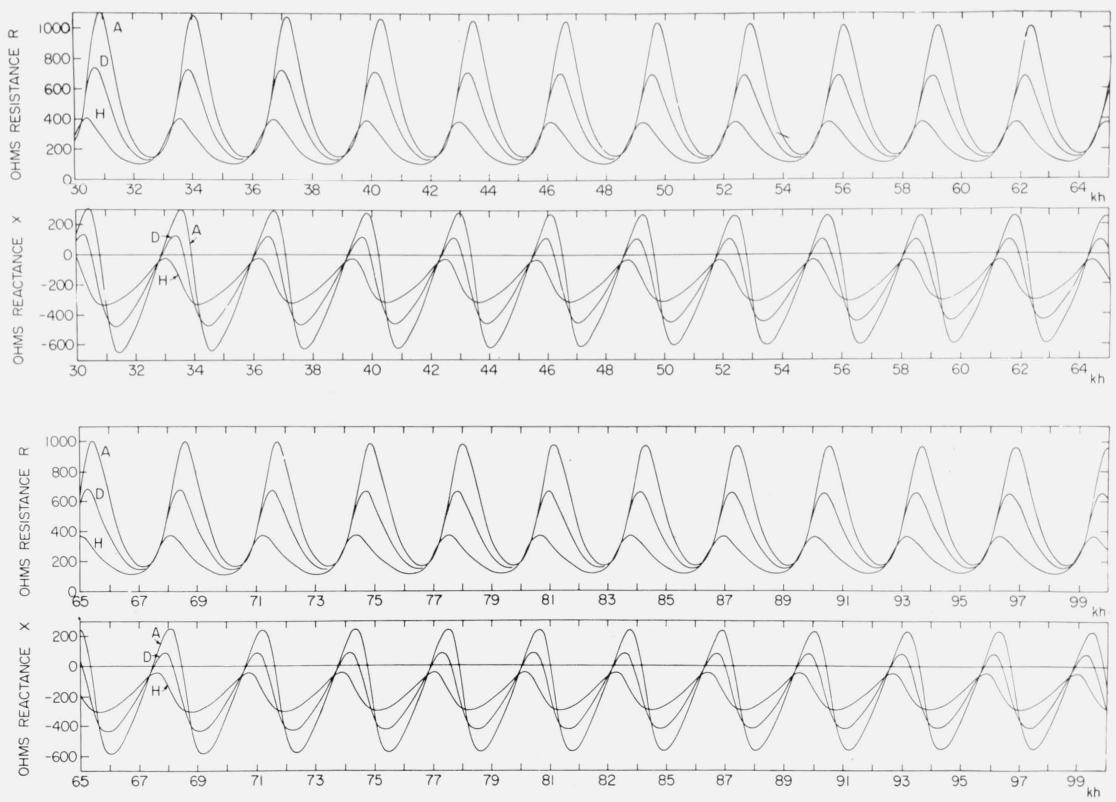

Fig. 4b. Resistance and reactance of long cylindrical antenna, Wu theory. $\mathrm{a} / \lambda=0.001191(\mathrm{~A}), 0.003175(\mathrm{D}), 0.008496(\mathrm{H})$ 


\section{References}

Gooch, D. W., C. W. Harrison, Jr., R. W. P. King, and T. T. Wu (Jan. 1962), Impedances and admittances of long antennas in air and in dissipative media with tables of the functions $f(p) \pm i g(p)=1 \pm i p$, Cruft Laboratory Tech. Rpt. No. 353.

Iizuka, K., R. W. P. King, and S. Prasad (1963), The admittance of a very long cylindrical antenna, Proc. IEE, 110, No. 2, 303-309

Iizuka, K., and R. W. P. King (1962), Terminal-zone corrections for a dipole driven by a two-wire line, J. Res. NBS 66D (Radio Prop.) No. 6, 775-781.

King, R. W. P. (1945), Electromagnetic Engineering, [1] appendix 2 (McGraw-Hill Book Co.). A Dover edition of this book will be available early in 1963 under the title Fundamental Electromagnetic Theory.

King, R. W. P. (1955a), Transmission Line Theory, pp. 405-411, 430-437 (McGraw-Hill Book Co., New York, N.Y.).

King, R. W. P. (April 1955b), End correction for a coaxial line when driven over a ground screen, Trans. IRE AP-3, $66-72$.

King, R. W. P. (1956), Theory of linear antennas, ch. II (Harvard University Press).

King, R. W. P., and C. W. Harrison, Jr. (1960), Half-wave cylindrical antenna in dissipative medium: Current and impedance, J. Res. NBS 64D (Radio Prop.) No. 4, 365-380.
King, R. W. P., C. W. Harrison, Jr., and David H. Denton, Jr. (1961), The electrically short antenna as a probe for measuring electron densities and collision frequencies in an ionized region, J. Res. NBS 65D (Radio Prop.) No. 4, $371-384$

King, R. W. P. (1962), Dipoles in dissipative media, pp. 199-241 in R. E. Langer, Electromagnetic waves (University of Wisconsin Press)

King, R. W. P., and H. J. Schmitt (May 1962), Transient response of linear antennas and loops, Trans. IRE AP-10, 222-228.

King, R. W. P., and K. Iizuka (1963), The complete electromagnetic field of dipoles in dissipative media, Trans. IRE AP-11.

Moore, R. K. (June 1951), Theory of radio communication between submerged submarines, Thesis, Cornell University.

Papas, C. H. (Sept. 1958), On the infinitely long cylindrical antenna, Cruft Laboratory Tech. Rpt. No. 58

$\mathrm{Wu}, \mathrm{T}$. T., and R. W. P. King (Jan. 1959), Driving point and input admittance of linear antennas, J. Appl. Phys. 30, $74-76$.

Wu, T. T. (1961). Theory of the dipole antenna and the twowire transmission line, J. Math. Phys. 2, 550-574.

(Paper 67D3-269) 